PROCEEDINGS OF THE

AMERICAN MATHEMATICAL SOCIETY

Volume 127, Number 1, January 1999, Pages 21-27

S 0002-9939(99)04740-1

\title{
LEVEL ONE REPRESENTATIONS OF $U_{q}\left(G_{2}^{(1)}\right)$
}

\author{
NAIHUAN JING
}

(Communicated by Roe Goodman)

\begin{abstract}
We construct a level one representation of the quantum affine algebra $U_{q}\left(G_{2}^{(1)}\right)$ by vertex operators from bosonic fields.
\end{abstract}

\section{INTRODUCTION}

Quantum affine algebras, the quantum groups associated to the affine KacMoody Lie algebras, provide an important underlying symmetry for the quantum Yang-Baxter equation [6] and quantum statistical models [11]. Explicit realizations of their representations are much needed in applications of quantum affine algebras. For instance, the Frenkel-Reshetikhin vertex operators [8] associated with the representations can be used to give solutions of the quantum Knizhnik-Zamolodchikov equation.

Lusztig first studied the abstract representations of quantum Kac-Moody algebras [20]. The program of constructing various representations was started in [7] for level one irreducible modules of ADE types, and subsequently twisted types were given in [12] and $B_{n}^{(1)}$ in [4]. Recently we have constructed symplectic quantum affine algebras in [17] for level one and in [16] for level $-1 / 2$. The case of $F_{4}^{(1)}$ can also be done similarly [15] using the idea of quantum $Z$-algebras [13, 15]. Besides the bosonic constructions, fermionic constructions were furnished in [10]. The $q$-Wakimoto construction was also known [21, 1, 22] afterwards. Other representations of classical quantum affine algebras have also been constructed [2]. The exceptional case of $G_{2}^{(1)}$ was the only case that has not been explicitly constructed.

The purpose of the paper is to give a explicit level one construction of the quantum affine algebra $U_{q}\left(G_{2}^{(1)}\right)$ by vertex operators. The idea of the construction follows that of quantum $Z$-algebras $[13,15]$, which is a $q$-deformation of the classical $(q=1) Z$-algebras $[19,18]$. We construct some auxiliary vertex operators for the short root. This is parallel to the known constructions of the affine Lie algebra $G_{2}^{(1)}$ $[5,9,23]$, though the specialization of $q=1$ in our construction is new even in the classical case.

Received by the editors April 30, 1997.

1991 Mathematics Subject Classification. Primary 17B37, 17 B67.

Key words and phrases. Quantum $Z$-operators, vertex operators.

This research was supported in part by NSA grants MDA904-96-1-0087 and MDA904-97-10062 .

(C)1999 American Mathematical Society 
The paper is organized as follows. In section two we review the quantum affine algebra $U_{q}\left(G_{2}^{(1)}\right)$. Section three gives the Fock space representation of the quantum affine algebra $U_{q}\left(G_{2}^{(1)}\right)$ stated in Theorem 3.1. Section four uses quantum vertex operator techniques to prove Theorem 3.1. In the proof of Serre relations we have to show a relation about certain symmetric functions, which is characteristic in the quantum affine algebras as noted in [12]. The Serre relations in $G_{2}^{(1)}$ turn out to be the most complicated one among both untwisted and twisted cases, and actually capture all the existed phenomena in other types.

\section{Quantum Affine Algebra $U_{q}\left(G_{2}^{(1)}\right)$}

Let $\alpha_{i}(i=1,2)$ be the simple roots of the simple Lie algebra $G_{2}$, and $\lambda_{i}$ be the fundamental weight. Let $P=\mathbf{Z} \lambda_{1}+\mathbf{Z} \lambda_{2}$ and $Q=\mathbf{Z} \alpha_{1}+\mathbf{Z} \alpha_{2}$ be the weight and root lattices. We then let $\Lambda_{i}, i \in I=\{0,1,2\}$, be the fundamental weights for the affine Lie algebra $G_{2}^{(1)}$, where $\Lambda_{i}=\lambda_{i}+\Lambda_{0}$, and $\lambda_{i}$ are the fundamental weights for the finite dimensional simple Lie algebra $G_{2}$. The nondegenerate symmetric bilinear form $(\mid)$ on $\mathbf{h}^{*}$ is given by

$$
\left(\alpha_{i} \mid \alpha_{j}\right)=d_{i} a_{i j}, \quad\left(\delta \mid \alpha_{i}\right)=(\delta \mid \delta)=0 \text { for all } i, j,
$$

where $\left(d_{0}, d_{1}, d_{3}\right)=(1,1,1 / 3)$ and $A=\left(a_{i j}\right)=\left(\begin{array}{cc}2 & -1 \\ -3 & 2\end{array}\right)$.

Let $q_{i}=q^{d_{i}}=q^{\frac{1}{2}\left(\alpha_{i} \mid \alpha_{i}\right)}, i \in I$. The quantum affine algebra $U_{q}\left(G_{2}^{(1)}\right)$ is the associative algebra with 1 over $\mathbf{C}\left(q^{1 / 6}\right)$ generated by the elements $x_{i k}^{ \pm}, a_{i l}, K_{i}^{ \pm 1}$, $\gamma^{ \pm 1 / 2}, q^{ \pm d}(i=1,2, \cdots, n, k \in \mathbf{Z}, l \in \mathbf{Z} \backslash\{0\})$ with the following defining relations $[6,3,14]$ :

$$
\begin{gathered}
{\left[\gamma^{ \pm 1 / 2}, u\right]=0 \text { for all } u \in \mathbf{U},} \\
{\left[a_{i k}, a_{j l}\right]=\delta_{k+l, 0} \frac{\left[\left(\alpha_{i} \mid \alpha_{j}\right) k\right]}{k} \frac{\gamma^{k}-\gamma^{-k}}{q-q^{-1}},} \\
{\left[a_{i k}, K_{j}^{ \pm 1}\right]=\left[q^{ \pm d}, K_{j}^{ \pm 1}\right]=0} \\
q^{d} x_{i k}^{ \pm} q^{-d}=q^{k} x_{i k}^{ \pm}, q^{d} a_{i l} q^{-d}=q^{l} a_{i l}, \\
K_{i} x_{j k}^{ \pm} K_{i}^{-1}=q^{ \pm\left(\alpha_{i} \mid \alpha_{j}\right)} x_{j k}^{ \pm}, \\
{\left[a_{i k}, x_{j l}^{ \pm}\right]= \pm \frac{\left[\left(\alpha_{i} \mid \alpha_{j}\right) k\right]}{k} \gamma^{\mp|k| / 2} x_{j, k+l}^{ \pm},} \\
\left(z-q^{ \pm\left(\alpha_{i} \mid \alpha_{j}\right)} w\right) X_{i}^{ \pm}(z) X_{j}^{ \pm}(w)+\left(w-q^{ \pm\left(\alpha_{i} \mid \alpha_{j}\right)} z\right) X_{j}^{ \pm}(w) X_{i}^{ \pm}(z)=0, \\
{\left[X_{i}^{+}(z), X_{j}^{-}(w)\right]=\frac{\delta_{i j}}{q_{i}-q_{i}^{-1}}\left(\psi_{i}\left(w \gamma^{1 / 2}\right) \delta\left(\frac{w \gamma}{z}\right)-\varphi_{i}\left(w \gamma^{-1 / 2}\right) \delta\left(\frac{w \gamma^{-1}}{z}\right)\right)}
\end{gathered}
$$

where $X_{i}^{ \pm}(z)=\sum_{n \in \mathbf{Z}} x_{i, n} z^{-n-1}, \psi_{i m}$ and $\varphi_{i m}\left(m \in \mathbf{Z}_{\geq 0}\right)$ are defined by

$$
\begin{aligned}
& \sum_{m=0}^{\infty} \psi_{i m} z^{-m}=K_{i} \exp \left(\left(q_{i}-q_{i}^{-1}\right) \sum_{k=1}^{\infty} a_{i k} z^{-k}\right) \\
& \sum_{m=0}^{\infty} \varphi_{i,-m} z^{m}=K_{i}^{-1} \exp \left(-\left(q_{i}-q_{i}^{-1}\right) \sum_{k=1}^{\infty} a_{i,-k} z^{k}\right)
\end{aligned}
$$




$$
\sum_{r=0, \sigma \in S_{m}}^{m=1-A_{i j}}(-1)^{r}\left[\begin{array}{c}
m \\
r
\end{array}\right]_{i} \sigma \cdot X_{i}^{ \pm}\left(z_{1}\right) \cdots x_{i}^{ \pm}\left(z_{r}\right) x_{j}^{ \pm}(w) x_{i}^{ \pm}\left(z_{r+1}\right) \cdots x_{i}^{ \pm}\left(z_{m}\right)=0,
$$

where the symmetric group $S_{m}$ acts on $z_{i}$ by permuting their indices.

\section{Fock SPACE REPRESEnTATiONS}

Let $a_{i}(m)(i=1,2)$ be the operators satisfying the Heisenberg relations for $U_{q}\left(G_{2}^{(1)}\right)$ at $\gamma=q$, and let $b(m)$ and $c(m)$ be two independent free bosonic operators with the relations

$$
\begin{aligned}
{\left[a_{i}(m), a_{j}(n)\right] } & =\delta_{m+l, 0} \frac{\left[\left(\alpha_{i} \mid \alpha_{j}\right) m\right]}{m}[m] \\
{[b(m), b(n)] } & =-\delta_{m+l, 0} \frac{[2 m / 3]}{m}[m] \\
{[c(m), c(n)] } & =\delta_{m+l, 0} \frac{[2 m / 3]}{m}[5 m / 3] \\
{\left[a_{i}(m), b(n)\right] } & =\left[a_{i}(m), c(n)\right]=[b(m), c(n)]=0 .
\end{aligned}
$$

Let $\beta_{2}$ be an auxiliary simple root isomorphic to $\alpha_{2}$. We define the Fock module $\mathcal{F}$ as the tensor product of the symmetric algebra generated by $a_{i}(-n), b(-n), c(-n)$ $(n \in \mathbb{N})$ and the twisted group algebra $\mathbb{C}\left\{P+\mathbb{Z} \beta_{2}\right\}$ generated by $e^{\alpha}, e^{\beta}$ subject to the relation

$$
e^{\alpha_{1}} e^{\alpha_{2}}=-e^{\alpha_{2}} e^{\alpha_{1}}, \quad e^{\alpha} e^{\beta}=e^{\beta} e^{\alpha}, \quad e^{\alpha} e^{\alpha}=e^{\alpha} e^{\alpha},
$$

where $\alpha \in P$, and $\beta$ is an element of the auxiliary lattice $\mathbb{Z} \alpha_{2}$ (another copy of the sublattice generated by the short root $\alpha_{2}$ ). In the following we reserve $\beta$ to denote an element from this auxiliary lattice.

The element 1 is the vacuum state. We define the action by

$$
a_{i}(n) \cdot 1=0 \quad(n>0), \quad b_{i}(n) \cdot 1=0 \quad(n>0),
$$

The elements $a_{i}(0)$ and $b(0)$ act as differential operators by

$$
a_{i}(0) e^{\alpha}=\left(\alpha_{i} \mid \alpha\right) e^{\alpha}, \quad b(0) e^{\beta}=-\frac{2}{3} e^{\beta} .
$$

As usual we define the normal product as the ordered product by moving annihilation operators $a_{i}(n), b(n), a_{i}(0), b(0)$ to the left.

Let's introduce the following operators:

$$
\begin{aligned}
& Y_{1}^{ \pm}(z)= \exp \left( \pm \sum_{n=1}^{\infty} \frac{a_{1}(-n)}{[n]} q^{\mp \frac{n}{2}} z^{n}\right) \exp \left(\mp \sum_{n=1}^{\infty} \frac{a_{1}(n)}{[n]} q^{\mp \frac{n}{2}} z^{-n}\right) e^{\alpha_{1}} z^{\mp a_{1}(0)} \\
& Y_{2}^{ \pm}(z)=\exp \left( \pm \sum_{n=1}^{\infty} \frac{a_{1}(-n)+b(-n)}{[n]} q^{\mp \frac{n}{2}} z^{n}\right) \exp \left(\mp \sum_{n=1}^{\infty} \frac{a_{2}(n)+b(n)}{[n]} q^{\mp \frac{n}{2}} z^{-n}\right) \\
& \cdot e^{\alpha+b} z^{\mp a_{2}(0)+b(0)}, \\
& U_{ \pm}(z)=\exp \left(\mp \sum_{n=1}^{\infty} \frac{[n / 3]}{[2 n / 3]} b( \pm n) z^{\mp n}\right) q^{\mp b(0) / 2} \\
& W_{ \pm}(z)=\exp \left(\mp \sum_{n=1}^{\infty} \frac{[n / 3]}{[2 n / 3]} c( \pm n) z^{\mp n}\right) .
\end{aligned}
$$


Theorem 3.1. The space $\mathcal{F}$ is a $U_{q}\left(G_{2}^{(1)}\right)$-module of level one under the action defined by $\gamma \longmapsto q, K_{i} \longmapsto q^{a_{i}(0)}, a_{i m} \longmapsto a_{i}(m), q^{d} \longmapsto q^{\bar{d}}$, and

$$
\begin{aligned}
& X_{1}^{ \pm}(z) \longmapsto Y_{1}^{ \pm}(z), \\
& X_{2}^{ \pm}(z) \longmapsto \frac{ \pm Y_{2}^{ \pm}(z)}{q_{2}-q_{2}^{-1}}\left(U_{ \pm}\left(q^{\mp 5 / 6} z\right) W_{ \pm}\left(q^{\mp 1 / 2} z\right)^{ \pm 1}-U_{ \pm}\left(q^{ \pm 5 / 6} z\right) W_{ \pm}\left(q^{ \pm 1 / 2} z\right)^{\mp 1}\right) .
\end{aligned}
$$

\section{Proof of the Theorem}

We now prove the theorem by checking that the action satisfies the Drinfeld relations. It is clear that $(2.2-2.6)$ are true by the construction. The relation (2.7) follows from the definition of $Y_{i}^{ \pm}(z)$ and the commutativity among $\alpha_{i}(n), b(n)$ and $c(n)$. So we only need to show (2.8) and (2.9).

We first compute the operator product expansions for $Y_{i}^{ \pm}(z)$ :

$$
\begin{aligned}
Y_{i}^{ \pm}(z) Y_{j}^{ \pm}(w) & =: Y_{i}^{ \pm}(z) Y_{j}^{ \pm}(w): \\
& \cdot \exp \left(-\sum_{n=1}^{\infty} \frac{\left[\left(\alpha_{i} \mid \alpha_{j}\right) n\right]}{n[n]} q^{\mp n}\left(\frac{w}{z}\right)^{n}\right) z^{\left(\alpha_{i} \mid \alpha_{j}\right)}, \\
Y_{i}^{ \pm}(z) Y_{j}^{\mp}(w) & =: Y_{i}^{ \pm}(z) Y_{j}^{\mp}(w): \\
& \cdot \exp \left(\sum_{n=1}^{\infty} \frac{\left[\left(\alpha_{i} \mid \alpha_{j}\right) n\right]}{n[n]}\left(\frac{w}{z}\right)^{n}\right) z^{-\left(\alpha_{i} \mid \alpha_{j}\right)} .
\end{aligned}
$$

For $\epsilon= \pm= \pm 1$ we define

$$
\begin{aligned}
& X_{2 \epsilon}^{+}(z)=Y_{2}^{+}(z) U_{\epsilon}\left(q^{-5 \epsilon / 6} z\right) W_{\epsilon}\left(q^{-1 \epsilon / 2} z\right), \\
& X_{2 \epsilon}^{-}(z)=Y_{2}^{-}(z) U_{\epsilon}\left(q^{5 \epsilon / 6} z\right) W_{\epsilon}\left(q^{1 \epsilon / 2} z\right)^{-1},
\end{aligned}
$$

so that $X_{2}^{ \pm}(z)=\frac{1}{q_{2}-q_{2}^{-1}}\left(X_{2+}^{ \pm}(z)-X_{2-}^{ \pm}(z)\right)$.

Note that for $i=j=1$ the relation (2.8) follows from the $\operatorname{sl}(2)$ case. For $\left(\alpha_{i} \mid \alpha_{j}\right)=-1$ (i.e. $i \neq j$ ) equations (4.1) become

$$
\begin{aligned}
& Y_{i}^{ \pm}(z) Y_{j}^{ \pm}(w)=: Y_{i}^{ \pm}(z) Y_{j}^{ \pm}(w):\left(z-q^{\mp 1} w\right)^{-1}, \\
& Y_{i}^{ \pm}(z) Y_{j}^{\mp}(w)=: Y_{i}^{ \pm}(z) Y_{j}^{\mp}(w):\left(z-q^{\mp 1} w\right),
\end{aligned}
$$

which implies that for $i \neq j$

$$
\begin{aligned}
& \left(z-q^{\mp 1} w\right) X_{i}^{ \pm}(z) X_{j}^{ \pm}(w)=\left(q^{\mp 1} z-w\right) X_{j}^{ \pm}(w) X_{i}^{ \pm}(z), \\
& {\left[X_{1}^{+}(z), X_{2}^{-}(w)\right]=0}
\end{aligned}
$$

where the latter is one case of relation (2.9).

To prove the remaining case of (2.8) we compute

$$
X_{2 \epsilon}^{+}(z) X_{2 \epsilon}^{+}(w)=: X_{2 \epsilon}^{+}(z) X_{2 \epsilon}^{+}(w): \frac{z-w}{z-q^{2 / 3} w} q^{(1+\epsilon) / 6}
$$

Then we immediately get the "+" case of relation $(2.8)$ for $i=j=2$. The "_" case is shown similarly.

In relation (2.9), again we only need to show the cases involved with the short root $\alpha_{2}$, since the proof of $\left[X_{1}^{+}(z), X_{1}^{-}(w)\right]$ is quite similar to that of type $\mathrm{A}$ in [12]. Observe that

$$
X_{2 \epsilon}^{+}(z) X_{2,-\epsilon}^{-}(w)=: X_{2 \epsilon}^{+}(z) X_{2,-\epsilon}^{-}(w):
$$


Thus we reduce the relation to the commutators $\left[X_{2 \epsilon}^{+}(z), X_{2 \epsilon}^{-}(w)\right]$. We compute that

$$
\begin{aligned}
& {\left[X_{2+}^{+}(z), X_{2+}^{-}(w)\right]} \\
& =: X_{2+}^{+}(z) X_{2+}^{-}(w):\left(\frac{z-q^{5 / 3} w}{z-q w} q^{-1 / 3}-\frac{w-q^{-5 / 3} z}{w-q^{-1} z} q^{1 / 3}\right) \\
& =: X_{2+}^{+}(z) X_{2+}^{-}(w): \frac{z-q^{5 / 3} w}{z} q^{-1 / 3} \delta\left(\frac{q w}{z}\right) \\
& =\left(q^{-1 / 3}-q^{1 / 3}\right) \psi_{2}\left(z q^{1 / 2}\right) \delta\left(\frac{q w}{z}\right)
\end{aligned}
$$

Similarly we can prove that

$$
\left[X_{2-}^{+}(z), X_{2-}^{-}(w)\right]=\left(q^{1 / 3}-q^{-1 / 3}\right) \phi_{2}\left(z q^{-1 / 2}\right) \delta\left(\frac{q^{-1} w}{z}\right) .
$$

Finally we use the quantum vertex operator calculus [12] to prove the Serre relations. The case $\left(a_{12}=-1\right)$ is similar to that of $U_{q}\left(A_{n}^{(1)}\right)$ [12]. We only check the other one $\left(a_{i j}=-3\right)$ in the "+" case:

$$
\begin{aligned}
\sum_{\sigma \in S_{4}} \sigma . & \left(X_{1}^{+}(w) X_{2}^{+}\left(z_{1}\right) X_{2}^{+}\left(z_{2}\right) X_{2}^{+}\left(z_{3}\right) X_{2}\left(z_{4}\right)\right. \\
- & {[4]_{2} X_{2}^{+}\left(z_{1}\right) X_{1}^{+}(w) X_{2}^{+}\left(z_{2}\right) X_{2}^{+}\left(z_{3}\right) X_{2}^{+}\left(z_{4}\right) } \\
+ & \frac{[4]_{2}[3]_{2}}{[2]_{2}} X_{2}^{+}\left(z_{1}\right) X_{2}^{+}\left(z_{2}\right) X_{1}^{+}(w) X_{2}^{+}\left(z_{3}\right) X_{2}^{+}\left(z_{4}\right) \\
- & {[4]_{2} X_{2}^{+}\left(z_{1}\right) X_{2}^{+}\left(z_{2}\right) X_{2}^{+}\left(z_{3}\right) X_{1}^{+}(w) X_{2}^{+}\left(z_{4}\right) } \\
& \left.+X_{2}^{+}\left(z_{1}\right) X_{2}^{+}\left(z_{2}\right) X_{2}^{+}\left(z_{3}\right) X_{2}\left(z_{4}\right) X_{1}^{+}(w)\right)=0
\end{aligned}
$$

Recalling that $X_{2}^{ \pm}(z)=\frac{1}{q_{2}-q_{2}^{-1}}\left(X_{2+}^{ \pm}(z)-X_{2-}^{ \pm}(z)\right)$ and using (4.1-4.6) and Wick's theorem, we can reduce the left-hand side of (4.8) to a linear combination of operator product terms : $X_{1}^{+}(w) X_{2 \epsilon_{1}}^{+}\left(z_{1}\right) X_{2 \epsilon_{2}}^{+}\left(z_{2}\right) X_{2 \epsilon_{3}}^{+}\left(z_{3}\right) X_{2 \epsilon_{4}}\left(z_{4}\right)$ :, where $\epsilon_{i}= \pm$. Thus the Serre relation is equivalently reduced to four Serre-like relations grouped by the number of appearances of $X_{2+}^{+}\left(z_{i}\right)$ in the product. Due to (4.7) the most complicated contraction functions comes from the case when all $\epsilon_{i}=+$. All four subcases can be treated similarly. In the following we will only prove the case when $\epsilon_{i}=+$. Ignoring the factor $\left(q_{2}-q_{2}^{-1}\right)^{-4}$, the left-hand side of this Serrelike relation is $q^{2}: X_{1}^{+}(w) X_{2+}^{+}\left(z_{1}\right) X_{2+}^{+}\left(z_{2}\right) X_{2+}^{+}\left(z_{3}\right) X_{2+}\left(z_{4}\right)$ : times the following expression:

$$
\begin{aligned}
& \sum_{\sigma \in S_{4}} \sigma \cdot \prod_{i} \frac{1}{\left(w-q^{-1} z_{i}\right)\left(z_{i}-q^{-1} w\right)} \prod_{i<j} \frac{z_{i}-z_{j}}{z_{i}-q^{2 / 3} z_{j}} \\
& \quad \cdot\left[\left(z_{1}-q^{-1} w\right) \cdots\left(z_{4}-q^{-1} w\right)+[4]_{2}\left(w-q^{-1} z_{1}\right)\left(z_{2}-q^{-1} w\right) \cdots\left(z_{4}-q^{-1} w\right)\right. \\
& \quad+\left[\begin{array}{c}
4 \\
2
\end{array}\right]_{2}\left(w-q^{-1} z_{1}\right)\left(w-q^{-1} z_{2}\right)\left(z_{3}-q^{-1} w\right)\left(z_{4}-q^{-1} w\right) \\
& \left.\quad+[4]_{2}\left(w-q^{-1} z_{1}\right) \cdots\left(w-q^{-1} z_{3}\right)\left(z_{4}-q^{-1} w\right)+\left(w-q^{-1} z_{1}\right) \cdots\left(w-q^{-1} z_{4}\right)\right],
\end{aligned}
$$

where the symmetric group $S_{4}$ acts on the ring of rational functions in $z_{i}$ by permutations on the indices. The $q$-binomial identity implies that the coefficients of 1 
and $w^{4}$ are zero, and the expression in the brackets is then simplified to

$$
\begin{aligned}
& q_{2}^{4}\left(q_{2}^{-6}-1\right)\left[q_{2}^{-1} w^{3}\left(q_{2}^{-12} z_{1}-q_{2}^{-6}\left(1+q_{2}^{-2}+q_{2}^{-4}\right) z_{2}+q_{2}^{-2}\left(1+q_{2}^{-2}+q_{2}^{-4}\right) z_{3}-z_{4}\right)\right. \\
& \quad+w^{2}\left(1+q_{2}^{-2}\right)\left(q_{2}^{-12} z_{1} z_{2}-q_{2}^{-6}\left(1+q_{2}^{-2}\right) z_{1} z_{3}+q_{2}^{-4}\left(1+q_{2}^{-2}+q_{2}^{-4}\right) z_{1} z_{4}\right. \\
& \left.\quad+q_{2}^{-4}\left(1+q_{2}^{-2}+q_{2}^{-4}\right) z_{2} z_{3}+q_{2}^{-4}\left(1+q_{2}^{-2}\right) z_{2} z_{4}-z_{3} z_{4}\right) \\
& \quad+q_{2}^{-1} w\left(q_{2}^{-12} z_{1} z_{2} z_{3}-q_{2}^{-6}\left(1+q_{2}^{-2}+q_{2}^{-4}\right) z_{1} z_{2} z_{3}\right. \\
& \left.\left.\quad+q_{2}^{-2}\left(1+q_{2}^{-2}+q_{2}^{-4}\right) z_{1} z_{3} z_{4}-z_{2} z_{3} z_{4}\right)\right] .
\end{aligned}
$$

Let $f\left(z_{1}, z_{2}, z_{3}, z_{4}\right)$ denote the above expression. Since $\prod_{i<j}\left(z_{i}-q_{2}^{2} z_{j}\right)\left(z_{i}-q_{2}^{-2} z_{j}\right)$ is symmetric, we see that the Serre relation is equivalent to the following identity:

$$
\sum_{\sigma \in S_{4}} \operatorname{sgn}(\sigma) \sigma\left(f\left(z_{1}, z_{2}, z_{3}, z_{4}\right) \prod_{i<j}\left(z_{i}-q_{2}^{-2} z_{j}\right)\right)=0 .
$$

We claim that (4.9) is true. Notice that it is enough to check only the coefficients of $w$ and $w^{2}$, and even these two are quite similar. The tedious checking of the coefficient of $w$ shows that it is zero indeed. Thus the Serre relation is proved.

The constructed level one representation is reducible due to the presence of auxiliary bosons $b(m)$ and $c(m)$. All integrable irreducible level one modules are contained in the Fock representation and can be recovered by the technique of the screening operators.

\section{REFERENCES}

[1] A. Abada, H. Bougourzi and M. El Gradechi, Deformations of Wakimoto representations, Mod. Phys. Lett. A8 (1993), 515-724. MR 94c:81065

[2] H. Awata, S. Odake, and J. Shiraishi, Free boson representation of $U_{q}\left(\hat{s l}{ }_{N}\right)$, Commun. Math. Phys. (1994), 61-83. MR 95g:81072

[3] J. Beck, Braid group action and quantum affine algebras, Commun. Math. Phys. 165 (1994), 555-568. MR 95i: 17011

[4] D. Bernard, Vertex operator representations of quantum affine algebras $U_{q}\left(B_{r}^{(1)}\right)$, Lett. Math. Phys. 17 (1989), 239-245. MR 90f: 17016

[5] D. Bernard and J. Thierry-Mieg, Level one representations od the simple affine Kac-Moody algebras in their homogeneous gradations, Commun. Math. Phys. 111 (1987), 181-246. MR 88m:17016

[6] V. G. Drinfeld, A new realization of Yangians and quantized affine algebras, Soviet Math. Dokl., 36 (1988), 212-216. MR 88j:17020

[7] I. B. Frenkel and N. Jing, Vertex representations of quantum affine algebras, Proc. Nat'l. Acad. Sci. USA 85 (1988), 9373-9377. MR 90e:17028

[8] I. Frenkel and Y. Reshetikhin, Quantum affine algebras and holomorphic difference equations, Commun. Math. Phys. 146, 1-60. MR 94c:17024

[9] P. Goddard, W. Nahm, D. I. Olive, and A. Schwimmer, Vertex operators for non-simply laced algebras, Commun. Math. Phys., 107 (1986), 179-212. MR 87k:17022

[10] H. Hayashi, Q-analogues of Clifford and Weyl algebras-spinor and oscillator representations of quantum affine algebras, Commun. Math. Phys. 127 (1990), 129-144. MR 91a:17015

[11] M. Jimbo and T. Miwa, Algebraic analysis of solvable lattice models, CBMS series 85, Amer. Math. Soc., Providence, RI, 1995. MR 96e:82037

[12] N. Jing, Twisted vertex representations of quantum affine algebras, Invent. Math. 102 (1990), 663-690. MR 92a:17019

[13] N. Jing, Higher level representations of the quantum affine algebra $U_{q}(\widehat{s l}(2))$, J. Algebra, 182 (1996), 448-468. MR 97f:17017

[14] N. Jing, On Drinfeld realization of quantum affine algebras, in Proc. of Conf. on the Monster and Lie Algebras (May, 1996), eds. by J. Ferrar and K. Harada, de Gruyter Verlag, Berlin, 1997, to appear; q-alg/9610035. 
[15] N. Jing, Quantum Z-algebras and representations of quantum affine algebras, to appear.

[16] N. Jing, Y. Koyama, and K. C. Misra, Bosonic realizations of $U_{q}\left(C_{n}^{(1)}\right)$, J. Algebra, to appear; q-alg/9701035.

[17] N. Jing, Y. Koyama, and K. C. Misra, Level one realizations of quantum affine algebras $U_{q}\left(C_{n}^{(1)}\right)$, to appear.

[18] J. Lepowsky and M. Primc, Standard modules for type one affine Lie algebras, Lect. Notes in Math., 1052, Springer-Verlag, New York, 1984, pp. 194-251. MR 86f:17015

[19] J. Lepowsky and R. L. Wilson, A new family of algebras underlying the Rogers-Ramanujan identities and generalizations, Proc. Nat'l. Acad. Sci. USA, 78 (1981), 7254-7258. MR 82k:10016

[20] G. Lusztig, Quantum deformations of certain simple modules over enveloping algebras, Adv. Math. 70 (1988), 237-249. MR 89k:17029

[21] A. Matsuo, A q-deformation of Wakimoto modules, primary fields and screening operators, Commun.Math.Phys. 160 (1994) 33-48. MR 95h:17033

[22] J. Shiraishi, Free boson representations $U_{q}\left(\widehat{s l}_{2}\right)$, Phys. Lett. A171 (1992), 243-248. MR 93k: 81108

[23] Y. Xu and C. Jiang, Vertex operators of $G_{2}^{(1)}$ and $B_{l}^{(1)}$, J. Phys. A: Gen. Math. 23 (1990), 3105-3121. MR 91j:17039

Department of Mathematics, North Carolina State University, Raleigh, North CarOLINA 27695-8205

E-mail address: jing@eos.ncsu.edu 